\title{
Why is Harvard Harvard?
}

\section{by Lizhen $\mathrm{Ji}^{*}$}

\section{Introduction}

Probably many people will agree that the USA has been one of the countries leading the world for a long time and will continue to do so in the coming years. American mathematics is also one of the driving forces behind the world of mathematics, and Harvard mathematics is both a part of the cause and outcome of this. Why is Harvard Harvard? What is so special or important about Harvard mathematics?

Obvious and fair questions! They are probably important and interesting questions too! There should be many books and articles addressing these questions already. But a complete answer from the firsthand experience is now given for the first time in the new book with an unusual title $A$ history in Sum by Steve Nadis and Shing-Tung Yau.

They recount how a lowly mathematics department became one of the world leaders, telling this inspiring story through the lives and works of people who called Harvard home.

Unlike a usual history book, this book contains real mathematics. Though the authors avoid equations and mathematics symbols, they do not shy away from real mathematical ideas.

They wrote this with the conviction that "good mathematicians (as well as good scientists in general) really need to understand their origins. By looking at the contributions of the great men and women from the past, we can trace a path showing how the important ideas in math evolved. And by looking at that path, we may gain helpful clues regarding avenues that are likely to be fruitful in the years ahead."

Clearly, this is a valuable book for mathematicians. But it is also a very interesting book for the general reader, since mathematicians are people too,

\footnotetext{
* Department of Mathematics, University of Michigan E-mail: lji@umich.edu
}

and some Harvard mathematical people had lived uniquely interesting lives. For mathematics students who want to have a glimpse of some major topics and players in modern mathematics and for mathematicians who want to have an informal, nontechnical descriptions of topics outside their specialities, this is also an informative and accessible book. The authors "aimed for something far broader than merely recounting the most notable success to have emerged from this department". Indeed, they tried to provide "a guide to a broad swath of modern mathematics, explaining concepts to nonspecialists that even mathematics students are not normally introduced to until graduate-level courses. Although lay readers will not be able to master these advanced subjects from our comparatively brief accounts, they can at least get a flavor of the work and perhaps get a gist of that it's about."

There have been many books on major breakthroughs in mathematics and stories behind them, especially in the past several years. Several recent books on mathematics departments and mathematicians connected to them have also appeared. For example, a book by C. Moore, Mathematics at Berkeley: $a$ history. A K Peters, Ltd., Wellesley, MA, 2007. 341 pp., was first one devoted the rise of a major mathematics department in USA, or in the world. It emphasizes the organizational aspects of the evolution of the department. A book by J. Segel, Recountings: Conversations with MIT Mathematicians, A K Peters, Ltd., Wellesley, MA, 2009. 330 pp., is more about lives of mathematicians at MIT in the format of interviews. A book by M. Cook, Mathematicians: an outer view of the inner world. Portraits by Mariana Cook. With an introduction by Robert Clifford Gunning and an afterword by Brandon Fradd. Princeton University Press, Princeton, NJ, 2009. 200 pp., gives a photographic and brief biographic description of some important mathemati- 
cians who were connected with Princeton at one point or another.

By comparison, the book by Nadis and Yau is more mathematical and explains the rise of Harvard through the lives and works of some major figures in mathematics. It is really an introduction to modern mathematics through Harvard people.

\section{A Glimpse of This Book}

It has been long time since I read a book from cover to cover. But when I picked up this book, I wanted to finish it.

Most new Ph.D.s in mathematics need to apply for postdoctoral positions, and one dream position is the Benjamin Peirce instructorship (or now Benjamin Peirce Fellowship). Of course, few people could get it. Since it is named after Benjamin Peirce, he must have played an important role in the history of Harvard mathematics. Probably few people bothered to find out more.

According to the book under review, he was the first person at Harvard who clinged to "the heretical notion that mathematicians ought to do original mathematics, which is to say, they should prove new theorems and solve problems that have never been solved before." It is also probably less known that he did his most important work in mathematics at the ripe age of 61 on associative algebras. The Peirce decomposition is a standard notion which is essential in describing the the boundary of symmetric cones. This work was also the first major contribution to mathematics by an American.

This book by Nadis and Yau contains much more information about Benjamin Peirce and his brilliant son Charles Sanders Peirce.

For analysts, a major prize from American Mathematics Society is the Bocher Memorial Prize. Who is Bocher? What was his connection with Harvard?

One of the most important theorems in complex analysis of one variable is the Riemann mapping theorem and is mentioned in almost every text book. But who was the person giving the first proof?

The answers to both questions are explained in a fascinating way in this book. Osgood proved this theorem, and he-together with Bocher-built the department of Mathematics at Harvard into a leading one in the USA: "They helped bring about the blossoming of mathematics nationwide by the example they set-helping to build a strong department at Harvard and also publishing first-rate research in journals based in the United States and abroad."

It is often an interesting question to ask who are the best pairs of father-son (or father-daughter) mathematicians. If there is any possible answer to this question, maybe this book can provide an answer to it too, among many other answers. ${ }^{1}$

But what did the senior Birkhoff do? Who were his students who left permanent marks on mathematics?

The USA is now a melting pot for international mathematicians from all corners of the world. But who was the first non-American professor hired permanently at Harvard? It was Ahlfors. It is probably less known that he pawned his Fields medal in order to escape the wartime Finland. This book describes the mathematical achievements of Ahlfors in some depth, especially his work leading to the first Fields medal in history, and also talks about what he had to go through to pursue this work during World War II.

Harvard was a famous center in algebraic geometry under the leadership of Zariski. Two of his students got Fields medals, and one received a Wolf prize. How did it happen? What was Zariski's reaction to the resolution of singularities by his student Hironaka? Zariski's main contribution probably lied in his work on the foundation of algebraic geometry rather than specific theorems. Indeed, accroding to the book, "Looking back, it seems pretty clear that Zariski's success in bolstering that foundation [of algebraic geometry] was probably more important in the long run than any individual theorem he proved." But Grothendieck made more fundamental changes to the foundation of algebraic geometry by introducing radical new ideas. What was Zariski's take on the new point of view of Grothendieck? One important new concept introduced by Grothendieck is étal cohomology. But how did it start? What's the contribution from Harvard people?

Some mathematicians are smart and fast, and some are slow and maybe profound. Harvard also provided such a closely related pair: Gleason and Mackey. What did they do?

How did number theory start at Harvard? Why are there so many things named after Tate?

Among all theorems in the history of mathematics, the theorem on classification of finite simple groups probably has the longest proof and is considered one of the most important results in the 20th century mathematics. Professors and students from Harvard were crucial in mapping out the whole program to finish it. Who were they?

If you have visited the Science Center at Harvard housing the mathematics department, you might be struck by its openness and ideal setting for interaction and communication, in sharp contrast with buildings at many other places. But which mathematician was partly responsible for this?

\footnotetext{
${ }^{1}$ Other candidates include E. Cartan and H. Cartan, E. Artin and M. Artin, M. Noether and E. Noether etc. Among all these pairs, the Birkhoff pair spent most of their careers at the same department.
} 
All these and many other questions are answered in this book. Once a great man said that if you want to know the flavor of a pear, take a bite. Read this book: you will be informed and entertained. You can see real people doing real mathematics. More importantly, you will understand why Harvard is Harvard! 